\title{
G

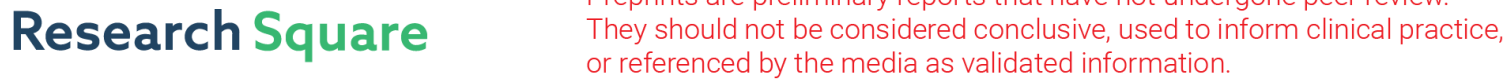 \\ Influence of post-thaw culture duration on \\ pregnancy outcomes in frozen blastocyst transfer cycles
}

\section{Xiufeng Ling ( $\square$ lingxiufeng_njfy@163.com )}

Women's Hospital of Nanjing Medical University, Nanjing Maternity and Child Health Care Hospital

Hui Ji

Women's Hospital of Nanjing Medical University, Nanjing Maternity and Child Health Care Hospital

\section{Shanren Cao}

Women's Hospital of Nanjing Medical University, Nanjing Maternity and Child Health Care Hospital

Hui Ding

Women's Hospital of Nanjing Medical University, Nanjing Maternity and Child Health Care Hospital

\section{Li Dong}

Women's Hospital of Nanjing Medical University, Nanjing Maternity and Child Health Care Hospital

\section{Chun Zhao}

Women's Hospital of Nanjing Medical University, Nanjing Maternity and Child Health Care Hospital

\section{Junqiang Zhang}

Women's Hospital of Nanjing Medical University, Nanjing Maternity and Child Health Care Hospital

Jing Lu

Women's Hospital of Nanjing Medical University, Nanjing Maternity and Child Health Care Hospital

\section{Xiuling Li}

Women's Hospital of Nanjing Medical University, Nanjing Maternity and Child Health Care Hospital

\section{Research Article}

Keywords: frozen blastocyst transfer, post-thaw culture duration, pregnancy outcome, propensity score matching

Posted Date: February 24th, 2022

DOI: https://doi.org/10.21203/rs.3.rs-1349549/v1

License: (1) (1) This work is licensed under a Creative Commons Attribution 4.0 International License. Read Full License 


\section{Abstract}

Background: To evaluate whether post-thaw culture duration affects clinical outcomes after frozen blastocyst transfer.

Methods: This retrospective cohort study included 3901 frozen-thawed blastocyst transfer cycles between September 2019 and March 2021. The cohorts were divided into two groups: developmental stage [day (D) 5 and D6] and culture duration after thawing (short culture of 2 to $6 \mathrm{~h}$ and long culture of 18 to $20 \mathrm{~h}$ ). Women in the short culture group following D6 blastocyst transfer were further assigned into three subgroups depending on the post-thaw culture period ( $2 \mathrm{~h}, 4 \mathrm{~h}$, and $6 \mathrm{~h})$. The main outcomes were the live birth rate (LBR), implantation rate (IR), clinical pregnancy rate (CPR), and abortion rate (AR). Propensity score matching (PSM) analysis was applied to ensure comparability within groups. Multivariable regression analysis was used to study the effect of post-thaw culture duration on pregnancy outcomes.

Results: The IR, CPR, AR, and LBR showed no statistical differences within the groups following D5 blastocyst transfer. Compared with the short culture group, patients in the long culture group had significantly lower IR (35.5 vs. $45.8 \%, P<0.001)$, CPR (45.3 vs. $56.6 \%, P=0.001)$, and LBR (35.5 vs. $48.5 \%, P<0.001)$, but a significantly higher AR $(21.6$ vs. $14.3 \%, P=0.049)$ following D6 blastocyst transfer. However, data failed to present the superiority of any short culture duration over another on the live birth outcome for embryos vitrified on D6 (aOR 0.96, 95\% Cl 0.53-1.73, $P=0.881$ for 4-h vs. 2-h subgroup; aOR 1.01, 95\% Cl 0.68-1.49, $P=0.974$ for 6-h vs. 2-h subgroup).

Conclusions: Both post-thaw protocols can be applied to patients with D5 blastocysts. To optimize the pregnancy outcome following D6 blastocyst transfer, the short culture duration is recommended. All three short culture durations ( $2 \mathrm{~h}, 4 \mathrm{~h}$, and $6 \mathrm{~h}$ ) can be applied, and the choice is based on each laboratory's workflow.

\section{Background}

With the development of the cryopreservation method of vitrification, the need of dealing with supernumerary embryos, and the objective of decreasing the ovarian hyperstimulation syndrome risk, frozen-thawed embryo transfer (FET) has become a commonly used method in various in vitro fertilization (IVF) treatments [1].

The selection criteria for the thawed embryos before transfer must be carefully planned, and they are rigorous to maximize pregnancy outcomes. Normally, embryos are transferred after a short culture duration, that is, after thawing from 1 to $6 \mathrm{~h}$ [2-4]. By contrast, some fertility centers use a long post-thaw culture interval (16 to $24 \mathrm{~h}$ ) [5-7]. One advantage for the different protocols is the flexibility of laboratory workflow; embryos can be thawed in the afternoon one day before transfer on a working day or thawed in the morning of a transfer day during weekends and holidays [8]. Moreover, the uterine cavity is an optimal "incubator", and in vitro embryo culture conditions cannot replicate fallopian tube and uterine 
environments in vivo. As such, some blastocysts fail to develop in vitro but they could have higher implantation rates in vivo [9]. Therefore, a short post-thaw culture protocol is more feasible. However, several publications have suggested that a short culture period might be insufficient to evaluate the developmental potential of blastocysts and that a long culture period could increase the degree of blastocoel expansion and provide more valuable information for the selection of embryos [10-13].

Presently, there is no consensus on which culture regimen yields the best pregnancy results. Some studies have suggested that the "post-thaw culture duration" should be considered as a confounding factor of FET outcomes $[9,14,15]$. One observational study indicated that a long-term incubation of $20 \mathrm{~h}$ could increase the implantation rate (IR) three-fold compared to a short-term incubation of $4 \mathrm{~h}$ [11]. However, other researchers have reported contradictory results; pregnancy outcomes were comparable for blastocysts thawed and cultured overnight and those thawed and transferred on the same day $[8,15]$. Nonetheless, their studies contained both day (D) 5 and D6 blastocysts, and conclusions could not be drawn for each embryo stage. Furthermore, Herbemont et al. [13] conducted a prospective randomized study that enrolled patients younger than 38 years of age with only good-quality D 5 blastocysts and demonstrated no difference in the IR, regardless of the culture duration. Nevertheless, there is no data on the suitability of D6 blastocysts for transfer, which represent a large proportion of blastocyst transfer cycles.

Therefore, we decided to evaluate the impact of post-thaw embryo culture on clinical outcomes after blastocyst transfer, not only of embryos developed on D5 but also of those developed on D6, to provide new insights for clinicians and embryologists facing warming protocols.

\section{Methods}

\section{Participants}

Participants who underwent frozen blastocyst transfers at the Reproductive Center of Women's Hospital of Nanjing Medical University, Nanjing, China (Nanjing Maternity and Child Health Care Hospital) between September 2019 and March 2021 were recruited. Patients younger than 43 years of age with at least one D5 or D6 blastocyst using their own eggs were eligible. The exclusion criteria were as follows: (a) presence of a uterine malformation; (b) cycles of oocyte donation, vitrified oocytes, or preimplantation genetic testing; (c) transfers of combined D5 and D6 blastocysts or D7 blastocysts; (d) maximal endometrial thickness (EMT) $<6 \mathrm{~mm}$; and (e) missing cycle data or follow-up.

\section{Ethical approval}

This retrospective cohort study was conducted according to the Declaration of Helsinki and approved by the Ethics Committee of Nanjing Maternity and Child Health Care Hospital (NJFY-2020-KY-070). Informed patient consent was not required as the study was retrospective in nature and analyzed patient data anonymously. 


\section{Stimulation and culture protocols}

The ovarian stimulation and embryo culture protocols used at our center have been previously published [16]. All patients were treated with the flexible gonadotropin-releasing hormone $(\mathrm{GnRH})$ antagonist protocol. Oocyte retrieval was scheduled 35 to $36 \mathrm{~h}$ after the injection of 10,000 IU human chorionic gonadotrophin (hCG, Livzon Pharmaceutical Group Inc., Zhuhai, China) to induce final oocyte maturation. After fresh transferring or freezing two cleavage-stage embryos with good morphology, all the supernumerary embryos were cultured until the blastocyst stage regardless of their quality.

\section{Vitrification and thawing procedures}

D5 or D6 blastocysts were scored according to the cavity expansion level, along with inner cell mass (ICM) and trophectoderm (TE) grading before freezing [17]. Blastocysts were selected for vitrification if they were at stage 3 or above with at least one B grade for either ICM or TE. We used a laser pulse (OCTAX laser shot, MTG Medical Technology, Altdorf, Germany) to artificially shrink the blastocoele before vitrification. The vitrification and thawing protocols, which have been previously described [18], followed the manufacturer's instructions (Kitazato BioPharma Co., Shizuoka, Japan). Blastocysts were placed into equilibration solution (7.5\% [v/v] ethylene glycol and 7.5\% [v/v] DMSO) at room temperature for 12-15 min and then transferred into vitrification solution (15\% [v/v] ethylene glycol, $15 \%$ [v/v] DMSO, and $0.5 \mathrm{M}$ sucrose) for $1 \mathrm{~min}$. Embryos were positioned at the top of the Cryotop and immediately frozen in liquid nitrogen for storage. During the thawing process, the cryotubes were immersed in thawing solution ( $1.0 \mathrm{M}$ sucrose) at $37^{\circ} \mathrm{C}$ for $1 \mathrm{~min}$. Subsequently, the blastocysts were transferred into diluent solution ( $0.5 \mathrm{M}$ sucrose) and incubated for $3 \mathrm{~min}$. Finally, the blastocysts were rinsed twice in washing solution at room temperature and then placed into $50 \mu \mathrm{L}$ of culture medium (G2; Vitrolife, Goteborg, Sweden), which was covered with mineral oil. Assisted hatching with laser treatment was conducted on thawed blastocysts, except for those with a 5 or 6 blastocoele re-expansion degree. Blastocysts were cultured at $37{ }^{\circ} \mathrm{C}$ in an atmosphere of $6 \% \mathrm{CO}_{2}, 5 \% \mathrm{O}_{2}$, and $89 \% \mathrm{~N}_{2}$. We evaluated the embryos half-anhour before transfer. Blastocyst survival was defined as partial or complete blastocoel re-expansion and $>50 \%$ intact cells. For blastocysts that had ceased to divide, an additional warming was performed.

Some blastocysts were thawed in the afternoon, indicating that there was an additional culture period of 18 to $20 \mathrm{~h}$ before transfer, while other blastocysts were thawed and transferred on the same day after a culture period of 2 to $6 \mathrm{~h}$. Participants were divided into two groups based on two different culture durations: the short culture group (a post-thaw culture duration of 2 to $6 \mathrm{~h}$ ) and the long culture group (a post-thaw culture duration of 18 to $20 \mathrm{~h}$ ). Good-quality blastocysts before vitrification were those with scores of $3 \mathrm{BB}$ or above (grade 3-6 AA/AB/BA/BB). Before transfer, re-expanded embryos with grades of $\geq 3 \mathrm{BB}$ in the short culture group and $\geq 5 \mathrm{BB}$ in the long culture group were considered good-quality blastocysts. 


\section{Endometrial preparation}

The endometrial preparation regimen was selected based on physician and patient preferences. Participants with regular menstruation were treated with the natural cycle (NC) protcol. Ovulation in the NC protocol was determined by monitoring follicular growth with transvaginal ultrasound and measuring the serum progesterone $(P)$ level. When the leading follicle was $\geq 18 \mathrm{~mm}$, the $P$ level was $\leq 1.5 \mathrm{ng} / \mathrm{ml}$, and the EMT was $\geq 6 \mathrm{~mm}, 10,000 \mathrm{IU}$ hCG was administered. Upon confirmation of ovulation by ultrasound, luteal phase support (LPS) was commenced, and patients were given $10 \mathrm{mg}$ of oral dydrogesterone (Abbott Biologicals B.V., Weesp, the Netherlands) three times daily $(\mathrm{P}+1)$. For the artificial cycles (AC), patients were given 4 to $6 \mathrm{mg}$ of oral estrogen (estradiol valerate, Progynova, DELPHARM Lille SAS., Lille, France) once daily starting on the second or third day of the menstrual cycle for one week, which was then adjusted to 6 to $10 \mathrm{mg}$ according to the EMT and serum estradiol $\left(E_{2}\right)$ level. After adequate endometrial proliferation and attainment of an EMT $\geq 6 \mathrm{~mm}$ and a serum $E_{2}$ concentration $\geq$ $200 \mathrm{pg} / \mathrm{ml}$, patients were administered $90 \mathrm{mg}$ of vaginal P (Crinone $8 \%$ gel, Fleet Laboratories Ltd., Watford, United Kingdom) once daily and $10 \mathrm{mg}$ of oral dydrogesterone three times daily $(\mathrm{P}+1)$. All embryo transfers were performed on day 6 of $P$ exposure $(P+6)$. If pregnancy was achieved, then LPS was continued until gestational weeks 10 to 12 .

\section{Pregnancy outcome}

Clinical pregnancy was confirmed as visualization of a gestational sac by ultrasound at 4 weeks after FET. Abortion was termed as fetal loss before 28 weeks of gestation. Live birth was defined as the delivery of a live-born infant beyond gestational week 28 . The IR was calculated from the number of intrauterine gestational sacs per number of transferred blastocysts. The primary outcome was the live birth rate (LBR). The secondary endpoints were the IR, clinical pregnancy rate (CPR), and abortion rate (AR).

\section{Statistical analysis}

All data were analyzed with SPSS 24.0 software (IBM, NY, USA). Continuous data with normal distribution were presented as mean \pm standard deviation (SD) and compared by the independent samples $t$ test, whereas continuous data with non-normal distribution were presented as median with interquartile range (IQR) and compared by the Mann-Whitney $U$ test. Categorical data were presented as count (percentage; $\%)$ and compared by the Pearson's chi-square test or Fisher's exact test.

To ensure comparability between patients with a short culture period and those with a long culture period, we implemented propensity score matching (PSM) analysis in D5 embryo transfer cycles. Patients were matched according to the propensity score with a 0.1 SD caliper and a ratio of 1:1. The following variables were included in the matching procedures: female age, infertility type, infertility duration, infertility cause, body mass index (BMI), basal follicle stimulating hormone (FSH) level, anti-Müllerian 
hormone (AMH) level, EMT, endometrial preparation protocol, number of transferred embryos, and number of good-quality embryos before transfer. Multivariable logistic regression analysis was performed to explore the effect of post-thaw culture duration on the live birth outcomes. Potential confounding factors showing clinical relevance or a $P$ value $<0.1$ in univariate analysis were included in the regression models. Statistical significance was accepted at $P<0.05$.

\section{Results}

\section{Study population}

Fig. 1 depicts our selection process. Of the initial 3901 FET cycles during the study period, 995 cycles were excluded. Overall, 2906 blastocyst embryo transfers met the eligibility criteria for analysis, including 2035 D5 blastocyst transfers and 871 D6 blastocyst transfers along with 950 short culture cycles and 1956 long culture cycles. We analyzed FET cycles in two distinct parts: part 1 follows D5 embryo transfers and part 2 follows D6 embryo transfers.

\section{Part 1: D5 blastocyst transfers}

The short culture group consisted of 406 cycles before PSM and 384 cycles after matching. The number of participants in the long culture group before and after PSM were 1629 and 384, respectively. The unpaired and paired baseline characteristics are presented in Table 1. The proportions of type, duration, cause of infertility, and endometrial preparation protocol, as well as female age, AMH level, and number of transferred embryos revealed significant differences within groups before PSM analysis $(P<0.05)$. After PSM, the demographics of the matched groups were comparable $(P>0.05)$.

The morphological characteristics of thawed blastocysts before vitrification and transfer are summarized in Table 2. When analyzing the embryo status before vitrification, there were no significant differences in blastocoel grade, the number of good-quality embryos, and the number of cycles with good-quality embryos between the two groups $(P>0.05)$. Surviving blastocysts were defined as those with partial or complete blastocoel re-expansion and the presence of $>50 \%$ intact cells. The survival rates in the short and long culture groups were similar ( $97.6 \mathrm{vs.} 97.4 \%, P=0.832)$. There was a significant difference in the degree of blastocoel expansion between the short and long culture groups (B5 and B6 stage: 26.2 vs. $95.7 \%, P<0.001)$.

Table 3 presents the FET outcomes after PSM. There were no significant differences between the two groups in terms of the IR (59.1 vs. $63.4 \%, P=0.170)$, CPR (63.8 vs. $66.9 \%, P=0.363)$, AR (11.8 vs. $14.4 \%$, $P=0.396)$, and LBR (56.3 vs. $57.3 \%, P=0.771)$.

The baseline characteristics of patients who had a live birth and those who did not are presented in Supplementary Table 1. With the post-thaw culture duration as the main exposure of interest, the covariate variables in the logistic regression analysis comprised of patient age, infertility type, serum 
levels of basal FSH and AMH, EMT, transferred embryo number, good-quality embryo number before transfer, and culture duration (short vs. long). The analysis revealed that the short culture protocol had no statistical difference on LBR compared with the long culture group (adjusted odds ratio (aOR) 0.94, 95\% confidence interval $(\mathrm{Cl}) 0.70-1.27 ; P=0.687)$.

\section{Part 2: D6 blastocyst transfers}

In total, 544 FET cycles in the short culture group and 327 cycles in the long culture group were enrolled in the analysis. There were no statistically significant differences in the baseline clinical parameters between the two groups $(P>0.05)$ (Table 4).

The morphological characteristics of blastocysts before vitrification and transfer are listed in Table 5. The blastocele and embryo quality were comparable between the groups before vitrification. There was no statistically significant difference in the survival rate between the two groups ( 97.8 vs. $97.2 \%, P=0.540$ ). As expected, after a longer culture period, the proportion of hatching (B5) or hatched (B6) blastocysts was significantly higher in the long culture group than that in the short culture group (grade B5 and B6: 82.1 vs. $16.6 \%, P<0.001)$, whereas the embryo quality was similar between the two groups.

Table 6 presents the clinical outcomes. Cycles with a short post-thaw culture period had a significantly higher IR (45.8 vs. $35.5 \%, P<0.001)$ and CPR (56.6 vs. $45.3 \%, P=0.001)$ compared to those with a long culture period. Meanwhile, a significantly lower AR was found in the short culture group (14.3 vs. $21.6 \%, P$ $=0.049)$ compared to the long culture group. Additionally, 264 patients $(48.5 \%)$ had live births after a post-thaw culture duration of 2 to $6 \mathrm{~h}$ compared with 116 patients (35.5\%) after a culture duration of 18 to $20 \mathrm{~h}(P<0.001)$.

The regression analysis demonstrated that the impact of culture duration on LBR remained significant in the prediction model (aOR 1.73, 95\% Cl 1.29-2.32; $P<0.001$ for the short vs. long culture group), which was adjusted for maternal age, infertility cause, the number of transferred embryos, the number of cycles with good-quality embryos before transfer, and culture duration (Supplementary Table 2).

Clearly, the short culture protocol had statistically better clinical outcomes compared with the long culture protocol. We further analyzed the influence of three different short culture durations on LBR $(2 \mathrm{~h}$ vs. $4 \mathrm{~h}$ vs. $6 \mathrm{~h}$ ). An initial cohort of 544 FET cycles was divided into two groups: the LB group (cycles reached live births) and the non-LB group (cycles failed to end with live births). As shown in Table 7, there was a statistically significant difference in the proportion of good-quality blastocyst transfers between the two groups $(P<0.001)$. Meanwhile, no statistically significant difference in culture duration $(P=0.901)$ was observed. Table 8 shows the logistic regression model for predicting live births. The summary effects of LBR in 4-h and 6-h subgroups versus 2-h subgroup were aOR $0.96(95 \% \mathrm{Cl} 0.53-1.73, P=0.881)$ and aOR 1.01 (95\% Cl 0.68-1.49, $P=0.974)$, respectively.

\section{Discussion}


The results of our study demonstrate that different post-thaw culture durations had similar clinical outcomes on blastocyst development on D5. However, the IR, CPR, and LBR after a short period of warming ( 2 to $6 \mathrm{~h}$ ) were statistically higher than those after a long period of warming (18 to $20 \mathrm{~h}$ ) in frozen-vitrified D6 embryo transfer cycles. In terms of LBR, no difference was noticed between three different short culture intervals ( $2 \mathrm{~h}, 4 \mathrm{~h}$, or $6 \mathrm{~h}$ ).

Currently, the subject of which post-thaw culture duration is best continues to be debated. In 2003, one retrospective study compared the effects of a short or a long period after warming ( $4 \mathrm{~h}$ vs. $20 \mathrm{~h}$ ) on the clinical outcomes of frozen blastocyst transfer cycles [11]. In contrast to our study, a longer culture interval increased the CPR three-fold (27.0 vs. $8.0 \%)$ and the IR four-fold (23.4 vs. $6.1 \%)$ after a 20 -h incubation period compared to a 4-h incubation period. However, applying the results of this study should be interpreted with caution, as there were some major flaws. In addition, a study which involved frozen embryo transfer cycles of D5, D6, and D7 blastocysts showed that pregnancy outcomes were comparable for blastocysts thawed and cultured overnight 1 day before transfer and those thawed and transferred on the same day [8]. In another study, Hwang et al. [15] enrolled patients having both vitrified D5 and D6 blastocysts for transfer and demonstrated that a 2 to $4 \mathrm{~h}$ culture interval yielded similar IR, CPR, AR, and LBR with a 20 to $24 \mathrm{~h}$ culture interval. These two studies collectively examined blastocysts from two different embryo developmental stages, neglecting the fact that D5 and D6 embryos may have metabolic or epigenetic differences, which can lead to some other conclusions [19]. Here, we found that D5 blastocysts could be transferred at any culture duration, with a preference for a short culture duration in D6 blastocyst transfer cycles. The discrepancy between the two previously published studies $[8,15]$ and our study may be related to the inclusion of embryos at different developmental stages. For instance, Hwang and colleagues reported that the majority ( 80\%) of enrolled cycles were D5 transfer cycles, with the remaining enrolled cycles consisting of D6 embryo transfer cycles [15].

Although the molecular mechanisms associated with our findings, namely that the transfer of D6 blastocysts after short-term culture is associated with better pregnancy outcomes compared to long-term culture, are unclear, they may be explained as follows. The synchronization of the embryo and the endometrium is paramount for successful clinical pregnancy, although it is important to note that the optimized embryo-endometrial synchrony is often vague and obscure. Blastocyst transfers are normally performed on the sixth day of $\mathrm{P}$ administration [20]. For blastocysts developed on D6, an additional $24 \mathrm{~h}$ culture period is present before vitrification compared with D 5 blastocysts. If embryo thawing is scheduled one day before transfer, another 16 to $24 \mathrm{~h}$ culture period will ensue, which might contribute to asynchrony between the embryo and the endometrium, leading to a decreased IR and a higher AR. Moreover, many events occur between thawing and hatching, such as blastocyst expansion, blastocoel collapse, zona pellucida thinning, and zona escape, which collectively can affect embryo development [21]. The prolonged culture time after warming can increase the degree of blastocoel expansion, indicating that a higher proportion of embryos can develop to stage 5 or $6[13,15]$. Marcos et al. [21] utilized a time-lapse system and observed that human blastocysts reaching stage 5 could experience one or more collapse-expansions of the blastocoel cavity. Their findings revealed that the collapse of blastocysts adversely affected the IR; the IRs for patients with collapsed blastocysts compared to those 
without collapsed embryos were $48.5 \%$ and $35.1 \%$, respectively. The collapse-expansion phenomenon was further confirmed in subsequent publications [22,23]. Single and multiple collapses occurred in $22 \%$ $(61 / 277)$ and $24 \%$ (66/277) of the examined blastocysts, respectively, and the live birth rate was significantly lower if multiple collapses occurred [22]. Meanwhile, blastocysts with spontaneous collapse in vitro were less likely to implant compared to embryos without collapse [23]. In our D6 embryo transfer cycles, $16.6 \%$ (130/781) of blastocysts reached the hatching or hatched stage before transfer in the short culture group compared to $82.1 \%$ (400/487) of blastocysts in the long culture group. Based on the available evidence, the incidence of collapse-expansion and multiple collapses is expected to be much higher after subjecting slowly developed D6 embryos to a longer post-thaw culture. We, therefore, postulate that poor transfer outcomes for D6 blastocyst transfers might be due to more mechanical stress and excessive energy consumption with regards to the repeated collapse-expansion events [21].

In addition to investigate that the short post-thaw duration results better pregnancy outcomes for D6 blastocysts, the best specific interval remains a debatable subject that would be meaningful for the laboratory practice. There were three short post-thaw culture durations of $2 \mathrm{~h}, 4 \mathrm{~h}$, and $6 \mathrm{~h}$ in our study. Multivariable logistic regression analysis revealed that the LBR did not differ, regardless of the culture duration. A review of the literature shows no clear agreement on the time of blastocele re-expansion after warming. In a study by Ahlstrom et al. [3], most of the blastocysts were assessed for re-expansion 1 to $5 \mathrm{~h}$ after warming, and the results indicated that the time had little contribution on the re-expansion degree once an interval of $2 \mathrm{~h}$ was reached. Hwang et al. [15] also reported that $90 \%$ of blastocysts completed reexpansion within an average of 1.4 to $3.5 \mathrm{~h}$ after warming. A longer in vitro culture protocol might increase metabolic or mechanical stress based on the already compromised D6 blastocyst viability. Compared with the 2-h culture group, the 6-h culture group showed no detrimental effects on the LBR (aOR $1.01,95 \% \mathrm{Cl} 0.68-1.49, P=0.974$ ), indicating that a culture period up to $6 \mathrm{~h}$ could still reliably ascertain embryo viability and developmental competency. Nonetheless, the sample size of this subgroup was relatively small for effective statistical analysis. Thus, further investigation in a large-scale clinical trial is warranted.

No statistically significant reduction in the LBR after long-term culture was observed for blastocysts vitrified on D5. Since the number of good-quality transferred embryos was equally distributed between the two groups, our results were in line with a recent study that enrolled 162 blastocysts transfer cycles with only good-quality D5 blastocysts [13]. The authors investigated whether the post-thaw culture duration (1 $\mathrm{h}$ vs. $18 \mathrm{~h}$ ) could influence the FET results. Their prospective randomized study demonstrated that the IR, $\mathrm{CPR}$, and AR were similar, regardless of the post-thaw culture duration. As such, both warming protocols can be applied to patients with good-quality D 5 blastocysts.

To date, several studies have suggested that there is a correlation between blastocyst developmental competence and a higher degree of blastocele re-expansion after thawing $[10,13,15]$. It is understandable, compared with the short duration group, that the long culture group showed a better ability to promote full expansion of blastoceles. As shown in a prospective study [13], the group with a post-thaw interval of 16 to $22 \mathrm{~h}$ had a higher proportion of B5/6 grade at the time of transfer compared 
with an interval of 0.5 to $5 \mathrm{~h}$ (38.6 vs. $12.7 \%, P<0.001)$. Additionally, B5/B6 grade blastocysts in the long culture protocol demonstrated a significantly higher IR than B4 grade blastocysts in the short culture protocol (52.9 vs. $32.9 \%, P=0.048)$. Consequently, the implantation potential of blastocysts cannot be always evaluated after a short post-thaw culture interval. Maezawa et al. [24] applied time-lapse imaging and revealed that blastocysts that failed to expand $5 \mathrm{~h}$ after thawing could not develop to the hatched stage and $25 \%$ of blastocysts remained shrunken after $6 \mathrm{~h}$. Thus, an overnight culture protocol in D5 blastocyst transfer cycles can not only select embryos with re-expansion, but also maintain their developmental ability until hatching. Currently, data have shown the priority of transferring D5 blastocysts to D6 blastocysts both in fresh $[25,26]$ and frozen $[2,27]$ embryo transfer cycles. The underlying reasons can be partly explained by better embryo quality [2], decreased abnormal spindle rate [28], and low aneuploidy incidence [29, 30]. All these reasons lead to the fact that blastocysts developed on D5 might have higher quality and improved implantation potential than D6 embryos to cope well with extended warming period. To conclude, the abovementioned reasons indicate that a long post-thaw culture period is harmless to the clinical outcomes of D5 blastocyst transfer cycles compared with a short post-thaw culture period.

To the best of our knowledge, the current study is the first to use a larger sample size to examine the effects of post-thaw culture duration on frozen blastocyst transfer outcomes involving both D5 and D6 embryos. A major strength of this study was that we evaluated both vitrified/warmed D5 and D6 blastocyst transfer cycles and observed that post-thaw culture duration plays an important role in predicting the LBR for D6 blastocysts. Our study also firstly examined frozen D6 blastocyst transfer outcomes from the viewpoint of three short culture strategies after warming. Data showed that LBR had a similar trend after a shorter culture regimen in D6 blastocyst transfer cycles, regardless of the post-thaw duration. Another strength was that PSM analysis was used to control for potential covariates, thereby ensuring that the outcomes were independent of the different patient characteristics in some way. Finally, our study was conducted in the past 1.5 years, which means that the laboratory conditions, culture media, and embryo selection criteria remained consistent. Given the undisputed high performance of vitrification compared with slow cooling protocols, the need of a longer culture period to allow for survival and additional morphology evaluation of thawed blastocysts seems non-existent. Some may argue that it is a common procedure to culture D6 blastocysts for a shorter post-thaw interval; however, there is a lack of consensus in the previous literature, and some laboratories continue to culture thawed D6 blastocysts for many hours for logistic reasons. Thus, we believe our study is still clinically relevant and worthy of interest.

Despite our efforts, there were some shortcomings in this study. Although PSM analysis was applied to match the confounders, this was a retrospective study and selection bias could not be excluded. Our results revealed that transferring D6 blastocysts after a short culture interval after thawing yielded better FET outcomes; however, we could not determine which specific interval was the best. A prospective randomized control study with a larger sample size is needed to confirm our results. 


\section{Conclusions}

In summary, the outcomes of short and long post-thaw culture durations did not differ in D5 blastocyst transfer cycles. Both embryo culture strategies can be applied and the decision is based on each center's work schedule. Nevertheless, for blastocysts vitrified on D6, a long culture period after thawing adversely affects clinical results. A short post-thaw culture protocol is recommended for patients with D6 blastocysts to optimize pregnancy outcomes. Apart from this, our data indicated that all three short durations achieved successful and comparable pregnancy outcomes that can be feasibly applied by each laboratory.

\section{Abbreviations}

LBR: Live birth rate; IR: Implantation rate; CPR: Clinical pregnancy rate; AR: Abortion rate; PSM: Propensity score matching; IVF: in vitro fertilization; FET: Frozen-thawed embryo transfer; EMT: Endometrial thickness; GnRH: Gonadotropin-releasing hormone; hCG: Human chorionic gonadotropin; ICM: Inner cell mass; TE: Trophectoderm; NC: Natural cycle; P: Progesterone; LPS: Luteal phase support; AC: Artificial cycles; E2: Estradiol; BMI: Body mass index; FSH: Basal follicle stimulating hormone; AMH: anti-Müllerian hormone; $\mathrm{Cl}$ : Confidence interval; OR: Odd ratio.

\section{Declarations}

\section{Ethics approval and consent to participate}

This study was performed in line with the principles of the Declaration of Helsinki. Approval was granted by the Ethics Committee of Nanjing Maternity and Child Health Care Hospital (NJFY-2020-KY-070). Informed patient consent was not required as the study was retrospective and analyzed patient data anonymously.

\section{Consent for publication}

Not applicable.

\section{Availability of data and materials}

The datasets analysed during the current study are not publicly available due to our ongoing research but are available from the corresponding author on reasonable request.

\section{Competing interests}

The authors declare that they have no competing interests. 


\section{Funding}

This work was supported by the National Natural Science Foundation of China [grant number 81701507, 81871210, 81971386]; Jiangsu Province "333" project; and the Open fund of State Key Laboratory of Reproductive Medicine, Nanjing Medical University [grant number SKLRM-K201806].

\section{Authors' contributions}

H.J. performed the analysis and wrote the manuscript. S.R.C. participated in the study design and drafted the article. H.D. and L.D. participated in the acquisition and analysis of data. C.Z., J.Q.Z., and J.L. reviewed the final article and made appropriate corrections and suggestions to improve it. X.L.L. and X.F.L. were the corresponding authors and participated in the study design, did the final proof reading and confirmed the final version. All authors read and approved the final manuscript.

\section{Acknowledgements}

The authors thank all the staff members of the Department of Reproductive Medicine of Women's Hospital of Nanjing Medical University for their support and cooperation.

\section{Authors' information}

${ }^{1}$ Department of Reproductive Medicine, Women's Hospital of Nanjing Medical University, Nanjing Maternity and Child Health Care Hospital, Nanjing, 210004, China

\section{References}

1. Roque M, Haahr T, Geber S, Esteves SC, Humaidan P. Fresh versus elective frozen embryo transfer in IVF/ICSI cycles: a systematic review and meta-analysis of reproductive outcomes. Hum Reprod Update. 2019;25(1):2-14. doi: 10.1093/humupd/dmy033.

2. Ferreux L, Bourdon M, Sallem A, Santulli P, Barraud-Lange V, Le Foll N, et al. Live birth rate following frozen-thawed blastocyst transfer is higher with blastocysts expanded on Day 5 than on Day 6 . Hum Reprod. 2018;33(3):390-8. doi: 10.1093/humrep/dey004.

3. Ahlstrom A, Westin C, Wikland M, Hardarson T. Prediction of live birth in frozen-thawed single blastocyst transfer cycles by pre-freeze and post-thaw morphology. Hum Reprod. 2013;28(5):1199209. doi: 10.1093/humrep/det054.

4. Tubbing A, Shaw-Jackson C, Ameye L, Colin J, Rozenberg S, Autin C. Increased live births after day 5 versus day 6 transfers of vitrified-warmed blastocysts. J Assist Reprod Genet. 2018;35(3):417-24. doi: 10.1007/s10815-017-1097-x. 
5. Yang H, Yang Q, Dai S, Li G, Jin H, Yao G, et al. Comparison of differences in development potentials between frozen-thawed D5 and D6 blastocysts and their relationship with pregnancy outcomes. J Assist Reprod Genet. 2016;33(7):865-72. doi: 10.1007/s10815-016-0712-6.

6. Haas J, Meriano J, Laskin C, Bentov Y, Barzilay E, Casper RF, et al. Clinical pregnancy rate following frozen embryo transfer is higher with blastocysts vitrified on day 5 than on day 6. J Assist Reprod Genet. 2016;33(12):1553-7. doi: 10.1007/s10815-016-0818-x.

7. Kang SM, Lee SW, Yoon SH, Kim JC, Lim JH, Lee SG. Comparison of clinical outcomes between single and double vitrified-warmed blastocyst embryo transfer according to the day of vitrification. J Assist Reprod Genet. 2013;30(6):779-85. doi: 10.1007/s10815-013-0017-y.

8. Fang C, Yue CM, Huang R, Wei LN, Jia L. Pregnancy outcomes of blastocysts cultured overnight after thawing. Arch Gynecol Obstet. 2016;293(6):1347-56. doi: 10.1007/s00404-016-4059-0.

9. Haas J, Meriano J, Bassil R, Barzilay E, Casper RF. Prolonged culture of blastocysts after thawing as a tool for improving prediction of success. J Assist Reprod Genet. 2018;35(12):2195-9. doi: 10.1007/s10815-018-1308-0.

10. Du QY, Wang EY, Huang Y, Guo XY, Xiong YJ, Yu YP, et al. Blastocoele expansion degree predicts live birth after single blastocyst transfer for fresh and vitrified/warmed single blastocyst transfer cycles. Fertil Steril. 2016;105(4):910-9 e1. doi: 10.1016/j.fertnstert.2015.12.014.

11. Guerif F, Cadoret V, Poindron J, Lansac J, Royere D. Overnight incubation improves selection of frozen-thawed blastocysts for transfer: preliminary study using supernumerary embryos. Theriogenology. 2003;60(8):1457-66. doi: 10.1016/s0093-691x(03)00130-4.

12. Minasi MG, Colasante A, Riccio T, Ruberti A, Casciani V, Scarselli F, et al. Correlation between aneuploidy, standard morphology evaluation and morphokinetic development in 1730 biopsied blastocysts: a consecutive case series study. Hum Reprod. 2016;31(10):2245-54. doi: 10.1093/humrep/dew183.

13. Herbemont C, Chekroune S, Bonan S, Cedrin-Durnerin I, Vivot A, Sonigo C, et al. Impact of postwarming culture duration on clinical outcomes of vitrified good-quality blastocyst transfers: a prospective randomized study. Fertil Steril. 2018;110(7):1290-7. doi:

10.1016/j.fertnstert.2018.07.1153.

14. Rato ML, Gouveia-Oliveira A, Plancha CE. Influence of post-thaw culture on the developmental potential of human frozen embryos. J Assist Reprod Genet. 2012;29(8):789-95. doi: 10.1007/s10815012-9793-z.

15. Hwang JY, Park JK, Kim TH, Eum JH, Song H, Kim JY, et al. The impact of post-warming culture duration on clinical outcomes of vitrified-warmed single blastocyst transfer cycles. Clin Exp Reprod Med. 2020;47(4):312-8. doi: 10.5653/cerm.2020.03832.

16. Ji H, Shi X, Wang J, Cao S, Ling X, Zhang J, et al. Peptidomic analysis of blastocyst culture medium and the effect of peptide derived from blastocyst culture medium on blastocyst formation and viability. Mol Reprod Dev. 2020;87(1):191-201. doi: 10.1002/mrd.23308. 
17. Gardner DK, Schoolcraft WB. Culture and transfer of human blastocysts. Curr Opin Obstet Gynecol. 1999;11(3):307-11. doi: 10.1097/00001703-199906000-00013.

18. Ji H, Zhou Y, Cao S, Zhang J, Ling X, Zhao C, et al. Effect of Embryo Developmental Stage, Morphological Grading, and Ploidy Status on Live Birth Rate in Frozen Cycles of Single Blastocyst Transfer. Reprod Sci. 2021;28(4):1079-91. doi: 10.1007/s43032-020-00381-6.

19. Irani M, O'Neill C, Palermo GD, Xu K, Zhang C, Qin X, et al. Blastocyst development rate influences implantation and live birth rates of similarly graded euploid blastocysts. Fertil Steril. 2018;110(1):95102 e1. doi: 10.1016/j.fertnstert.2018.03.032.

20. Mackens S, Santos-Ribeiro S, van de Vijver A, Racca A, Van Landuyt L, Tournaye H, et al. Frozen embryo transfer: a review on the optimal endometrial preparation and timing. Hum Reprod. 2017;32(11):2234-42. doi: 10.1093/humrep/dex285.

21. Marcos J, Perez-Albala S, Mifsud A, Molla M, Landeras J, Meseguer M. Collapse of blastocysts is strongly related to lower implantation success: a time-lapse study. Hum Reprod. 2015;30(11):2501-8. doi: 10.1093/humrep/dev216.

22. Bodri D, Sugimoto T, Yao Serna J, Kawachiya S, Kato R, Matsumoto T. Blastocyst collapse is not an independent predictor of reduced live birth: a time-lapse study. Fertil Steril. 2016;105(6):1476-83 e3. doi: 10.1016/j.fertnstert.2016.02.014.

23. Sciorio R, Herrer Saura R, Thong KJ, Esbert Algam M, Pickering SJ, Meseguer M. Blastocyst collapse as an embryo marker of low implantation potential: a time-lapse multicentre study. Zygote. 2020:1-9. doi: 10.1017/S0967199419000819.

24. Maezawa T, Yamanaka M, Hashimoto S, Amo A, Ohgaki A, Nakaoka Y, et al. Possible selection of viable human blastocysts after vitrification by monitoring morphological changes. J Assist Reprod Genet. 2014;31(8):1099-104. doi: 10.1007/s10815-014-0260-x.

25. Ozgur K, Berkkanoglu M, Bulut H, Humaidan P, Coetzee K. Perinatal outcomes after fresh versus vitrified-warmed blastocyst transfer: retrospective analysis. Fertil Steril. 2015;104(4):899-907 e3. doi: 10.1016/j.fertnstert.2015.06.031.

26. Franasiak JM, Forman EJ, Patounakis G, Hong KH, Werner MD, Upham KM, et al. Investigating the impact of the timing of blastulation on implantation: management of embryo-endometrial synchrony improves outcomes. Hum Reprod Open. 2018;2018(4):hoy022. doi: 10.1093/hropen/hoy022.

27. Bourdon M, Pocate-Cheriet K, Finet de Bantel A, Grzegorczyk-Martin V, Amar Hoffet A, Arbo E, et al. Day 5 versus Day 6 blastocyst transfers: a systematic review and meta-analysis of clinical outcomes. Hum Reprod. 2019;34(10):1948-64. doi: 10.1093/humrep/dez163.

28. Hashimoto S, Amo A, Hama S, Ito K, Nakaoka Y, Morimoto Y. Growth retardation in human blastocysts increases the incidence of abnormal spindles and decreases implantation potential after vitrification. Hum Reprod. 2013;28(6):1528-35. doi: 10.1093/humrep/det059.

29. Hernandez-Nieto C, Lee JA, Slifkin R, Sandler B, Copperman AB, Flisser E. What is the reproductive potential of day 7 euploid embryos? Hum Reprod. 2019;34(9):1697-706. doi:

10.1093/humrep/dez129.

Page 14/26 
30. Tiegs AW, Sun L, Patounakis G, Scott RT. Worth the wait? Day 7 blastocysts have lower euploidy rates but similar sustained implantation rates as Day 5 and Day 6 blastocysts. Hum Reprod. 2019;34(9):1632-9. doi: 10.1093/humrep/dez138.

\section{Tables}

\section{Table 1}

Patient demographic characteristics of day 5 blastocyst transfer before and after propensity score matching. 
Characteristic

Age (years)

Type of infertility, $\mathrm{n}(\%)$

Primary

Secondary

(

Secondary

Duration of infertility, n (\%)

$$
\leq 2
$$

3-5

$\geq 6$

Cause of infertility, n (\%)

female factor

male factor

combined factor

unknown factor

BMI $\left(\mathrm{kg} / \mathrm{m}^{2}\right)$

Basal FSH (mIU/mL)

$\mathrm{AMH}(\mathrm{ng} / \mathrm{ml})^{\mathrm{a}}$

Endometrial thickness

(mm)

Endometrial preparation

protocol, n (\%)
Before PSM

After PSM

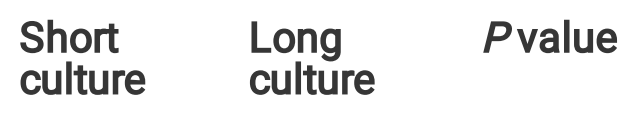

$(n=406) \quad(n=1629)$

$\begin{array}{lll}30.9 \pm & 30.4 \pm & 0.008 \\ 4.0 & 4.0 & \end{array}$

$<0.001$

874

(53.7)

755

(46.3)

(67.2)

133
$(32.8)$

273

(67.2)

(4)

(

$\begin{array}{lll}\text { Short } & \text { Long } & P \text { value } \\ \text { culture } & \text { culture } & \end{array}$

( $n=384) \quad(n=384)$

$\begin{array}{lll}30.9 \pm & 31.0 \pm & 0.655 \\ 4.0 & 4.0 & \end{array}$

$\begin{array}{ll}30.9 \pm & 31.0 \\ 4.0 & 4.0\end{array}$

0.368

\begin{tabular}{ll}
133 & 145 \\
$(34.6)$ & $(37.8)$ \\
\hline 251 & 239 \\
$(65.4)$ & $(62.2)$
\end{tabular}

0.001

0.869

$218 \quad 215$

(56.8) (56.0)

$\begin{array}{ll}128 & 134 \\ (33.3) & (34.9)\end{array}$

38 (9.9) $\quad 35(9.1)$

0.945

$<0.001$

947

(58.1)

(52.0)

74 (18.2)

183

(11.2)

$88(21.7)$

431

(26.5)

$33(8.1) \quad 68(4.2)$

$\begin{array}{ll}22.4 \pm & 22.6 \pm \\ 3.0 & 3.5\end{array}$

$7.5 \pm 1.8 \quad 7.4 \pm 2.0 \quad 0.518$

0.109

$5.1(3.2-\quad 0.010$

$4.4(2.7-$

7.4)

8.0)

$9.5 \pm 1.7 \quad 9.6 \pm 1.8 \quad 0.105$

0.022

$207 \quad 201$

(53.9) (52.3)

$66 \quad 72$

(17.2) (18.8)

$87 \quad 88$

(22.7) (22.9)

24 (6.3) $23(6.0)$

$\begin{array}{lll}22.3 \pm & 22.4 \pm & 0.850 \\ 3.0 & 3.4\end{array}$

$\begin{array}{lll}7.5 \pm & 7.5 \pm & 0.812\end{array}$

$\begin{array}{lll}4.4 & 4.9 & 0.158\end{array}$

$\begin{array}{ll}(2.7- & (3.1- \\ 7.4) & 8.0)\end{array}$

$9.5 \pm \quad 9.4 \pm$

$\begin{array}{ll}9.7 & 1.8\end{array}$

1.8

0.900

0.111 


\begin{tabular}{lllllll} 
NC & $92(22.7)$ & 461 & & 85 & 104 \\
& & $(28.3)$ & & $(22.1)$ & $(27.1)$ & \\
\hline AC & 314 & 1168 & & 299 & 280 & \\
& $(77.3)$ & $(71.7)$ & & $(77.9)$ & $(72.9)$ & \\
\hline $\begin{array}{l}\text { Number of transferred } \\
\text { embryos }\end{array}$ & $1.3 \pm 0.4$ & $1.6 \pm 0.5$ & $<0.001$ & $1.3 \pm$ & $1.3 \pm$ & 0.806 \\
\hline
\end{tabular}

Note: ${ }^{a}$ Data are presented as median (first quartile, third quartile). Other data are presented as mean \pm SD or $\mathrm{n}(\%)$. PSM, propensity score matching; $\mathrm{BMI}$, body mass index; $\mathrm{FSH}$, follicle stimulating hormone; $\mathrm{AMH}$, anti-Müllerian hormone; NC, natural cycle; AC, artificial cycle.

Table 2

Morphology of matched D5 blastocysts in each group before vitrification and transfer. 
Before vitrification

\begin{tabular}{|c|c|c|c|c|c|c|}
\hline Characteristic & $\begin{array}{l}\text { Short } \\
\text { culture }\end{array}$ & $\begin{array}{l}\text { Long } \\
\text { culture }\end{array}$ & $P$ value & $\begin{array}{l}\text { Short } \\
\text { culture }\end{array}$ & $\begin{array}{l}\text { Long } \\
\text { culture }\end{array}$ & $P$ value \\
\hline & $(n=384)$ & $(n=384)$ & & $(n=384)$ & $(n=384)$ & \\
\hline Warmed blastocysts (n) & 498 & 496 & & - & - & \\
\hline $\begin{array}{l}\text { Transferred blastocysts } \\
\text { (n) }\end{array}$ & - & - & & 486 & 483 & \\
\hline Survival rate, $\mathrm{n} / \mathrm{N}(\%)$ & - & - & & $\begin{array}{l}486 / 498 \\
(97.6)\end{array}$ & $\begin{array}{l}483 / 496 \\
(97.4)\end{array}$ & 0.832 \\
\hline Blastocoel grade, $\mathrm{n}(\%)$ & & & 0.155 & & & $<0.001$ \\
\hline 3 & $\begin{array}{l}112 \\
(22.5)\end{array}$ & $\begin{array}{l}136 \\
(27.4)\end{array}$ & & $35(7.2)$ & $2(0.4)$ & \\
\hline 4 & $\begin{array}{l}377 \\
(75.7)\end{array}$ & $\begin{array}{l}354 \\
(71.4)\end{array}$ & & $\begin{array}{l}324 \\
(66.7)\end{array}$ & $19(3.9)$ & \\
\hline 5 & $9(1.8)$ & $6(1.2)$ & & $\begin{array}{l}97 \\
(20.0)\end{array}$ & $\begin{array}{l}136 \\
(28.2)\end{array}$ & \\
\hline 6 & 0 & 0 & & $30(6.2)$ & $\begin{array}{l}326 \\
(67.5)\end{array}$ & \\
\hline $\begin{array}{l}\text { Good-quality blastocysts, } \\
\mathrm{n} / \mathrm{N}(\%)\end{array}$ & $\begin{array}{l}374 / 498 \\
(75.1)\end{array}$ & $\begin{array}{l}389 / 496 \\
(78.4)\end{array}$ & 0.214 & $\begin{array}{l}366 / 486 \\
(75.3)\end{array}$ & $\begin{array}{l}361 / 483 \\
(74.7)\end{array}$ & 0.838 \\
\hline $\begin{array}{l}\text { Cycles with good-quality } \\
\text { embryo number, } \mathrm{n}(\%)\end{array}$ & & & 0.785 & & & 0.941 \\
\hline 0 & $65(16.9)$ & $60(15.6)$ & & $\begin{array}{l}69 \\
(18.0)\end{array}$ & $\begin{array}{l}71 \\
(18.5)\end{array}$ & \\
\hline 1 & $\begin{array}{l}265 \\
(69.0)\end{array}$ & $\begin{array}{l}260 \\
(67.7)\end{array}$ & & $\begin{array}{l}264 \\
(68.8)\end{array}$ & $\begin{array}{l}265 \\
(69.0)\end{array}$ & \\
\hline 2 & $53(13.8)$ & $63(16.4)$ & & $\begin{array}{l}51 \\
(13.3)\end{array}$ & $\begin{array}{l}48 \\
(12.5)\end{array}$ & \\
\hline 3 & $1(0.3)$ & $1(0.3)$ & & 0 & 0 & \\
\hline
\end{tabular}

Table 3
Before transfer

$(n=384) \quad(n=384)$

$<0.001$

$324 \quad 19(3.9)$

326

$\begin{array}{ll}361 / 483 & 0.838\end{array}$

0.941
Cycles with good-quality embryo number, $n$ (\%)

Table 3

Clinical outcomes of the matched post-thaw culture duration after day 5 blastocysts transfer cycles. 


\begin{tabular}{llll}
\hline \multicolumn{1}{c}{ After PSM } & & \\
\hline Outcome & Short culture & Long culture & P value \\
\hline & $(\mathbf{n = 3 8 4 )}$ & $(\mathbf{n = 3 8 4 )}$ & \\
\hline Implantation rate, $\mathrm{n} / \mathrm{N}(\%)$ & $287 / 486(59.1)$ & $306 / 483(63.4)$ & 0.170 \\
\hline Clinical pregnancy rate, n/N (\%) & $245 / 384(63.8)$ & $257 / 384(66.9)$ & 0.363 \\
\hline Abortion rate, $\mathrm{n} / \mathrm{N}(\%)$ & $29 / 245(11.8)$ & $37 / 257(14.4)$ & 0.396 \\
\hline Live birth rate, $\mathrm{n} / \mathrm{N}(\%)$ & $216 / 384(56.3)$ & $220 / 384(57.3)$ & 0.771
\end{tabular}

Table 4 Patient demographic characteristics of day 6 blastocyst transfer. 


\begin{tabular}{|c|c|c|c|}
\hline \multirow[t]{2}{*}{ Characteristic } & Short culture & Long culture & \multirow[t]{2}{*}{$P$ value } \\
\hline & $(n=544)$ & $(n=327)$ & \\
\hline Age (years) & $31.2 \pm 4.1$ & $31.3 \pm 4.1$ & 0.672 \\
\hline Type of infertility, n (\%) & & & 0.651 \\
\hline Primary & $236(43.4)$ & $147(45.0)$ & \\
\hline Secondary & $308(56.6)$ & $180(55.0)$ & \\
\hline Duration of infertility, $\mathrm{n}(\%)$ & & & 0.766 \\
\hline$\leq 2$ & $268(49.3)$ & $155(47.4)$ & \\
\hline $3-5$ & $188(34.6)$ & $121(37.0)$ & \\
\hline$\geq 6$ & $88(16.2)$ & $51(15.6)$ & \\
\hline Cause of infertility, n (\%) & & & 0.784 \\
\hline female factor & $275(50.6)$ & $172(52.6)$ & \\
\hline male factor & $112(20.6)$ & $65(19.9)$ & \\
\hline combined factor & $120(22.1)$ & $73(22.3)$ & \\
\hline unknown factor & $37(6.8)$ & $17(5.2)$ & \\
\hline $\mathrm{BMI}\left(\mathrm{kg} / \mathrm{m}^{2}\right)$ & $22.3 \pm 3.1$ & $22.3 \pm 3.2$ & 0.848 \\
\hline Basal FSH (mIU/mL) & $7.5 \pm 1.9$ & $7.6 \pm 2.3$ & 0.499 \\
\hline $\mathrm{AMH}(\mathrm{ng} / \mathrm{ml})^{\mathrm{a}}$ & $3.9(2.3-6.5)$ & $4.2(2.5-6.8)$ & 0.176 \\
\hline Endometrial thickness (mm) & $9.4 \pm 1.8$ & $9.5 \pm 1.7$ & 0.564 \\
\hline Endometrial preparation protocol, n (\%) & & & 0.231 \\
\hline NC & $155(28.5)$ & $81(24.8)$ & \\
\hline AC & $389(71.5)$ & $246(75.2)$ & \\
\hline Number of transferred embryos & $1.4 \pm 0.5$ & $1.5 \pm 0.5$ & 0.125 \\
\hline
\end{tabular}

Table 5

Morphology of D6 blastocysts in each group before vitrification and transfer. 
Before vitrification

\begin{tabular}{|c|c|c|c|c|c|c|}
\hline Characteristic & $\begin{array}{l}\text { Short } \\
\text { culture }\end{array}$ & $\begin{array}{l}\text { Long } \\
\text { culture }\end{array}$ & $P$ value & $\begin{array}{l}\text { Short } \\
\text { culture }\end{array}$ & $\begin{array}{l}\text { Long } \\
\text { culture }\end{array}$ & $P$ value \\
\hline & $(n=544)$ & $(n=327)$ & & $(n=544)$ & $(n=327)$ & \\
\hline Warmed blastocysts (n) & 799 & 501 & & - & - & \\
\hline $\begin{array}{l}\text { Transferred blastocysts } \\
\text { (n) }\end{array}$ & - & - & & 781 & 487 & \\
\hline Survival rate, $\mathrm{n} / \mathrm{N}(\%)$ & - & - & & $\begin{array}{l}781 / 799 \\
(97.8)\end{array}$ & $\begin{array}{l}487 / 501 \\
(97.2)\end{array}$ & 0.540 \\
\hline Blastocoel grade, n (\%) & & & 0.192 & & & $<0.001$ \\
\hline 3 & $\begin{array}{l}138 \\
(17.3)\end{array}$ & $84(16.8)$ & & $59(7.6)$ & $10(2.1)$ & \\
\hline 4 & $\begin{array}{l}601 \\
(75.2)\end{array}$ & $\begin{array}{l}394 \\
(78.6)\end{array}$ & & $\begin{array}{l}592 \\
(75.8)\end{array}$ & $\begin{array}{l}77 \\
(15.8)\end{array}$ & \\
\hline 5 & $34(4.3)$ & $12(2.4)$ & & $68(8.7)$ & $\begin{array}{l}156 \\
(32.0)\end{array}$ & \\
\hline 6 & $26(3.3)$ & $11(2.2)$ & & $62(7.9)$ & $\begin{array}{l}244 \\
(50.1)\end{array}$ & \\
\hline $\begin{array}{l}\text { Good-quality blastocysts, } \\
\text { n/N (\%) }\end{array}$ & $\begin{array}{l}320 / 799 \\
(40.1)\end{array}$ & $\begin{array}{l}182 / 501 \\
(36.3)\end{array}$ & 0.180 & $\begin{array}{l}319 / 781 \\
(40.9)\end{array}$ & $\begin{array}{l}178 / 487 \\
(36.6)\end{array}$ & 0.128 \\
\hline $\begin{array}{l}\text { Cycles with good-quality } \\
\text { embryo number, } n(\%)\end{array}$ & & & 0.641 & & & 0.087 \\
\hline 0 & $\begin{array}{l}269 \\
(49.4)\end{array}$ & $\begin{array}{l}172 \\
(52.6)\end{array}$ & & $\begin{array}{l}271 \\
(49.8)\end{array}$ & $\begin{array}{l}182 \\
(55.7)\end{array}$ & \\
\hline 1 & $\begin{array}{l}230 \\
(42.3)\end{array}$ & $\begin{array}{l}128 \\
(39.1)\end{array}$ & & $\begin{array}{l}227 \\
(41.7)\end{array}$ & $\begin{array}{l}112 \\
(34.3)\end{array}$ & \\
\hline 2 & $45(8.3)$ & $27(8.3)$ & & $46(8.5)$ & $\begin{array}{l}33 \\
(10.1)\end{array}$ & \\
\hline
\end{tabular}

Table 6

Before transfer

781

$781 / 799$

$487 / 501 \quad 0.540$

$<0.001$

Blastocoel grade, $n(\%)$

$$
138
$$

601

394

(78.6)

(75.8)

(15.8)

(32.0)

(50.1)

Good-quality blastocysts,

(40.1)

(36.3)

$319 / 781$

$178 / 487$

(36.6)

0.128

0.087

Cycles with good-quality embryo number, $n$ (\%)

Clinical outcomes of two post-thaw culture durations after day 6 blastocysts transfer cycles. 


\begin{tabular}{llll}
\multirow{2}{*}{ Outcome } & Short culture & Long culture & \multirow{2}{*}{ P value } \\
\cline { 2 - 3 } & $(\mathbf{n = 5 4 4 )}$ & $(\mathbf{n = 3 2 7 )}$ & \\
\hline Implantation rate, $\mathrm{n} / \mathrm{N}(\%)$ & $358 / 781(45.8)$ & $173 / 487(35.5)$ & $<0.001$ \\
\hline Clinical pregnancy rate, $\mathrm{n} / \mathrm{N}(\%)$ & $308 / 544(56.6)$ & $148 / 327(45.3)$ & 0.001 \\
\hline Abortion rate, $\mathrm{n} / \mathrm{N}(\%)$ & $44 / 308(14.3)$ & $32 / 148(21.6)$ & 0.049 \\
\hline Live birth rate, $\mathrm{n} / \mathrm{N}(\%)$ & $264 / 544(48.5)$ & $116 / 327(35.5)$ & $<0.001$
\end{tabular}

Table 7

Patient characteristics of day 6 transfer cycles reaching or failing to have live births after the short culture protocol. 


\begin{tabular}{|c|c|c|c|}
\hline \multirow[t]{2}{*}{ Characteristic } & LB & Non-LB & \multirow[t]{2}{*}{$P$ value } \\
\hline & $(n=264)$ & $(n=280)$ & \\
\hline Age (years) & $31.0 \pm 4.0$ & $31.3 \pm 4.2$ & 0.368 \\
\hline Type of infertility, n (\%) & & & 0.799 \\
\hline Primary & $116(43.9)$ & $120(42.9)$ & \\
\hline Secondary & $148(56.1)$ & $160(57.1)$ & \\
\hline Duration of infertility, n (\%) & & & 0.681 \\
\hline$\leq 2$ & $135(51.1)$ & $133(47.5)$ & \\
\hline $3-5$ & $87(33.0)$ & $101(36.1)$ & \\
\hline$\geq 6$ & $42(15.9)$ & $46(16.4)$ & \\
\hline Cause of infertility, n (\%) & & & 0.266 \\
\hline female factor & $130(49.2)$ & $145(51.8)$ & \\
\hline male factor & $63(23.9)$ & $49(17.5)$ & \\
\hline combined factor & $56(21.2)$ & $64(22.9)$ & \\
\hline unknown factor & $15(5.7)$ & $22(7.9)$ & \\
\hline $\mathrm{BMI}\left(\mathrm{kg} / \mathrm{m}^{2}\right)$ & $22.5 \pm 3.1$ & $22.2 \pm 3.0$ & 0.360 \\
\hline Basal FSH (mIU/mL) & $7.4 \pm 2.0$ & $7.6 \pm 1.8$ & 0.241 \\
\hline $\mathrm{AMH}(\mathrm{ng} / \mathrm{ml})$ & $4.1(2.5-6.7)$ & $3.8(2.1-6.4)$ & 0.217 \\
\hline Endometrial thickness (mm) & $9.5 \pm 1.9$ & $9.3 \pm 1.7$ & 0.123 \\
\hline Endometrial preparation protocol, $\mathrm{n}(\%)$ & & & 0.967 \\
\hline NC & $75(28.4)$ & $80(28.6)$ & \\
\hline AC & $189(71.6)$ & $200(71.4)$ & \\
\hline Number of transferred embryos & $1.5 \pm 0.5$ & $1.4 \pm 0.5$ & 0.121 \\
\hline Good-quality embryo number before transfer & & & $<0.001$ \\
\hline 0 & $103(39.0)$ & $168(60.0)$ & \\
\hline 1 & $133(50.4)$ & $94(33.6)$ & \\
\hline 2 & $28(10.6)$ & $18(6.4)$ & \\
\hline Culture duration & & & 0.901 \\
\hline $2 \mathrm{~h}$ & $139(52.7)$ & $144(51.4)$ & \\
\hline
\end{tabular}




\begin{tabular}{lll}
$4 \mathrm{~h}$ & $28(10.6)$ & $28(10.0)$ \\
\hline $6 \mathrm{~h}$ & $97(36.7)$ & $108(38.6)$
\end{tabular}

Note: LB, live birth.

\section{Table 8}

Multivariable logistic regression analysis on the correlation between three short culture durations and live births in day 6 blastocyst transfer cycles

\begin{tabular}{llll} 
Parameters & aOR & $95 \%$ Cl & $P$ value \\
\hline Age & 0.99 & $0.94-1.03$ & 0.503 \\
\hline BMI & 1.02 & $0.96-1.08$ & 0.474 \\
\hline Endometrial thickness & 1.07 & $0.97-1.18$ & 0.183 \\
\hline Number of transferred embryos & 1.18 & $0.79-1.76$ & 0.414 \\
\hline Good-quality embryo number before transfer & & & \\
\hline 0 & Reference & & \\
\hline 1 & 2.37 & $1.65-3.42$ & $<0.001$ \\
\hline 2 & 2.22 & $1.13-4.39$ & 0.021 \\
\hline Culture duration & & & \\
\hline 2h & Reference & & \\
\hline 4h & 0.96 & $0.53-1.73$ & 0.881 \\
\hline $6 h$ & 1.01 & $0.68-1.49$ & 0.974
\end{tabular}

Note: aOR, adjusted odds ratio; $\mathrm{Cl}$, confidence interval.

\section{Figures}




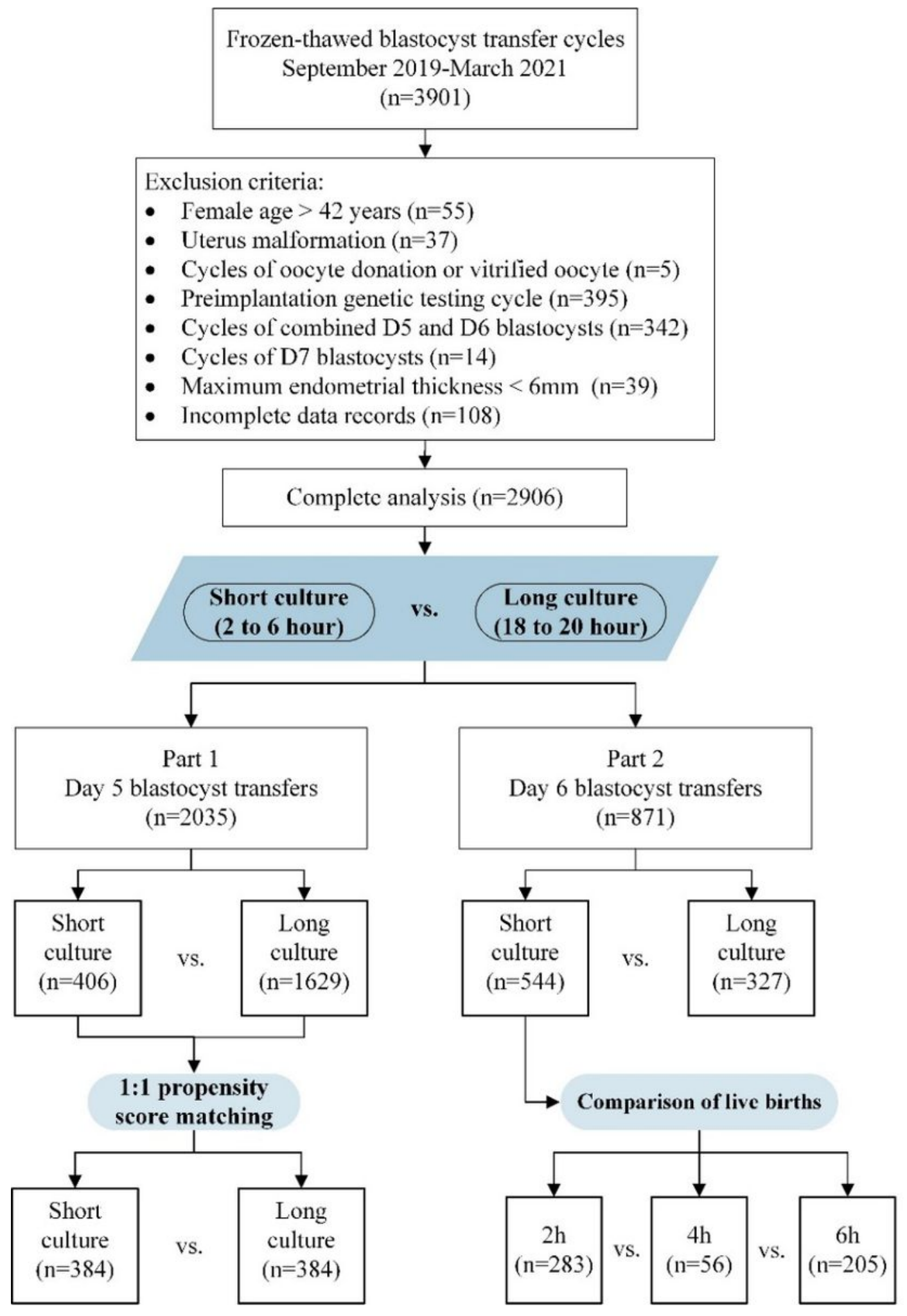

Figure 1

Flow chart.

\section{Supplementary Files}

This is a list of supplementary files associated with this preprint. Click to download. 
- Supplementaryinformation.docx

Page 26/ 26 